\title{
Molecular Gas and Dust in Nearby Galactic Centers: from SMA to ALMA
}

\author{
Kazushi Sakamoto \\ Academia Sinica, Institute of Astronomy and Astrophysics \\ Taipei 10617, Taiwan \\ email: ksakamoto@asiaa.sinica.edu.tw
}

\begin{abstract}
SMA observations of molecular gas and dust in nearby galactic centers are presented to showcase remarkable ISM properties including warm to very hot gas, molecular clouds producing massive star clusters, and mechanical feedback through bubbles and outflows.
\end{abstract}

Keywords. galaxies: ISM — galaxies: nuclei — galaxies: active

\section{Introduction}

Molecular gas, dust, and star formation in galaxies often have their distributions peaked at the galactic centers (GCs). For their detailed study one needs to target multiple GCs among nearby galaxies with observing techniques providing high spatial resolutions. Submillimeter interferometry is useful for this study and in particular for the GCs that host significant star formation or other activities. This is because submillimeter molecular lines and dust continuum emission are enhanced in their hot environment.

The Submillimeter Array (SMA) has been a powerful tool for GC studies. It has been in operation since 2003 with capabilities including subarcsecond resolution imaging at $\lambda=1300-400 \mu \mathrm{m}$, wide-band spectral coverage, and better access to the southern sky. Not only is it a powerful telescope on its own, but it also is a pathfinder for the Atacama Millimeter-Submillimeter Array (ALMA). I present below some highlights of the SMA observations of molecular gas and dust in nearby, relatively active galactic centers. A glimpse of ALMA observations of one of them is also provided. These observations show remarkable gas properties such as warm to very hot gas and dust, massive molecular cloud complexes producing massive young star clusters, and feedback from the GC activities to the ISM through bubbles and outflows.

\section{Moderately Active GCs: NGC 253, NGC 1365, and M83}

These three galaxies have infrared luminosities in the range of $10^{10}-10^{11} L_{\odot}$, stellar bars, and significant concentrations of molecular gas called 'central molecular zones' (CMZ). These nearby CMZs are good targets to compare with the CMZ of our Galaxy, which is also a barred spiral galaxy.

The CMZs of NGC 253 and Milky Way are compared in Fig. 1 in the same emission line of $\mathrm{CO}(2-1)$ and at the same linear resolution of $20 \mathrm{pc}$. NGC 253 is easy to compare with our Galaxy because both are (nearly) edge-on. The two CMZs appear strikingly similar at the first sight. Both have an extent of about $500 \times 100 \mathrm{pc}^{2}$ and contain several prominent $\mathrm{CO}$ emission peaks such as the Sgr. B molecular cloud complex in the Galactic center. The CO peaks in both CMZs generally contain dense molecular gas traced with $\mathrm{HCN}$ and $\mathrm{HCO}^{+}$and host active star formation. At the same time, there are also obvious observational differences between the two CMZs. Both the peak integrated intensities and peak brightness temperatures are about a factor of three higher in the star-forming 

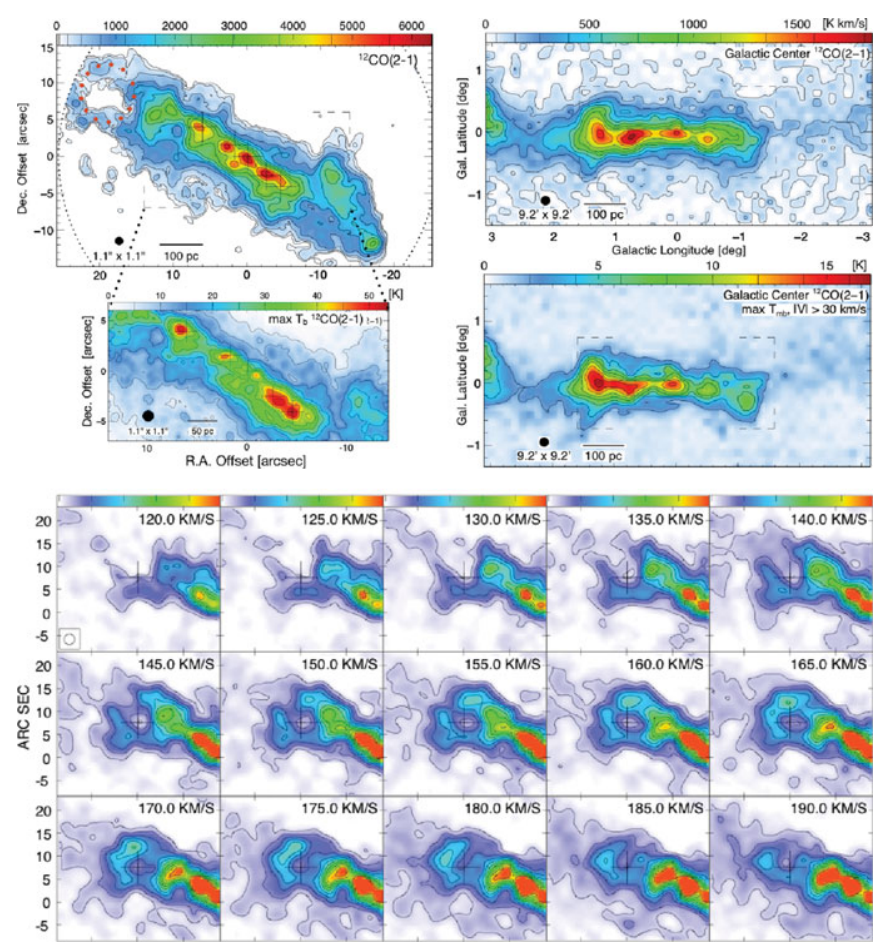

Figure 1. (Top) The central molecular zones of NGC 253 on the left and our Galaxy on the right. Integrated intensity maps and peak brightness temperature maps of $\mathrm{CO}(2-1)$ are shown at the same linear resolution of 20 pc. (Bottom) A 100 pc cavity in the CMZ of NGC 253 seen in $\mathrm{CO}(2-1)$ channel maps. Its location is marked with red dotted circle in the integrated intensity map above. These data are from Sakamoto et al. (2011).

molecular cloud complexes in NGC 253 than those in the center of Milky Way. For example the peak $T_{\mathrm{b}}$ in NGC 253 is about $50 \mathrm{~K}$ while it is $17 \mathrm{~K}$ in our CMZ in the matched data shown here. Another notable feature in the CMZ of NGC 253, without a counterpart in the Milky Way center, is a large cavity of molecular gas also shown in Fig. 1. Its extent is about $100 \mathrm{pc}$ by $100 \mathrm{~km} \mathrm{~s}^{-1}$. Modeled as an expanding superbubble, it has an estimated kinetic age and energy on the orders of $1 \mathrm{Myr}$ and $10^{46} \mathrm{~J}$, respectively (Sakamoto et al. 2006a). About 100 supernovae, maybe in a young massive cluster, may have created it. (Our Galaxy has smaller cavities and bubbles and so does NGC 253.)

While the morphological similarities of the two CMZs are probably due to a common bar-driven gas dynamics, the differences we saw between them are presumably a mixture of the causes and results of different star-forming luminosities in the two CMZs. The CMZ of NGC 253 is two orders of magnitude more infrared luminous than the CMZ of our Galaxy. Important questions we face are the root cause of this difference, as Dr. C. Henkel asked at the Symposium, and the mechanisms that connect the root cause to the actual difference in star formation. A likely root cause is the different total amounts of molecular gas in the two CMZs of similar sizes. The CMZ of NGC 253 has an order of magnitude larger CO luminosity than ours. Hence both the gas mass and star formation efficiency are ten times higher in NGC 253 if the same CO-to- $\mathrm{H}_{2}$ conversion factor applies to both CMZs. A possible mechanism for the enhanced star formation rate and efficiency in NGC 253 is the positive star formation feedback such as shock-induced star formation in the molecular bubbles. Another possible mechanism is an inherent non-linearity in the star formation law, e.g., due to star formation triggered by cloud collisions. 


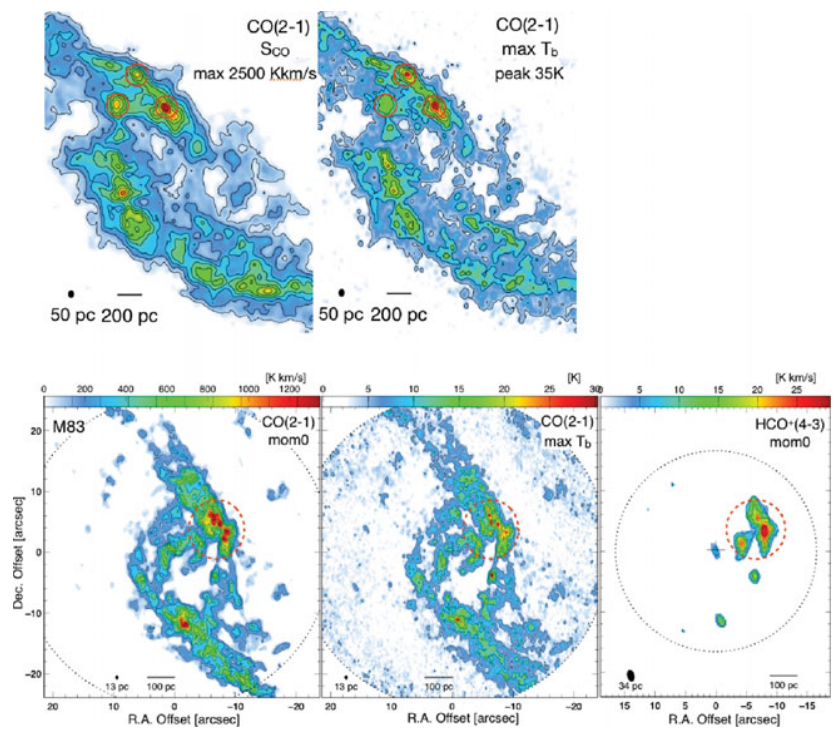

Figure 2. (Top) The central molecular zone of NGC 1365 in $\mathrm{CO}(2-1)$. Circles mark the locations of the three most prominent young massive clusters in the galactic center. (Bottom) The central molecular zone of $\mathrm{M} 83$ in $\mathrm{CO}(2-1)$ and $\mathrm{HCO}^{+}(4-3)$. The latter traces denser molecular gas than the former. The most active star-forming region is marked with a dotted circle.

The CMZs of NGC 1365 and M83 shown in Fig. 2 can be regarded as face-on counterparts of the edge-on CMZs in NGC 253 and our Galaxy. They clearly show circumnuclear molecular rings or spirals due to bar-driven gas dynamics, which are only inferred (e.g., from the position-velocity diagrams) in edge-on CMZs. In addition, these CMZs have massive, dense, and star-forming molecular cloud complexes similar to the ones in the CMZ of NGC 253 and our Galaxy.

NGC 1365 has in its CMZ three particularly luminous and young clusters and we found their natal molecular cloud complexes. The clusters are several Myr old and have masses and luminosities on the orders of $10^{5}-10^{6} M_{\odot}$ and $10^{9} L_{\odot}$, respectively (Galliano et al. 2012). The molecular cloud complexes associated with them have high brightness temperatures of $>30 \mathrm{~K}$ and high surface densities of $\geqslant 10^{3} M_{\odot} \mathrm{pc}^{-2}$ at a $50 \mathrm{pc}$ resolution (Sakamoto et al. 2007 and this work). These $\mathrm{CO}(2-1)$ properties are comparable to those at the molecular cloud complexes in the CMZ of NGC 253 for the same 50 pc resolution. The mass of molecular gas in such a 50 pc cloud complex is on the order of $10^{7} M_{\odot}$. See Elmegreen et al. (2009) for their plausible formation mechanisms.

M83 also shows, at the 'twin peaks' positions, concentrations of warm, dense, and highsurface density molecular gas. The northwestern peak is more prominent in the molecular line emission and also in terms of active star formation. Our spatial resolution toward M83 is $13 \mathrm{pc}$ in $\mathrm{CO}(2-1)$ and the data show structures at the size scale of giant molecular clouds (GMCs), i.e., 10 - several 10 pc. The most prominent CO peaks at this resolution have peak CO integrated intensities above $1000 \mathrm{~K} \mathrm{~km} \mathrm{~s}^{-1}$. Therefore these GMCs have molecular gas mean surface densities above $10^{3} M_{\odot} \mathrm{pc}^{-2}$ and violate Larson's third law on a near-universal column density of molecular clouds (Larson 1981), unless the COto- $\mathrm{H}_{2}$ conversion factor there is 30 times or more lower than the Galactic value of a few $M_{\odot} \mathrm{pc}^{-2}\left(\mathrm{~K} \mathrm{~km} \mathrm{~s}^{-1}\right)^{-1}$. In either case, this is an example of extreme gas properties in the CMZ of a moderately active (luminous) galaxy. Such gas property is not limited to the CMZ of M83. All galactic centers shown in this paper have CO integrated intensity peaks in (often large) excess of $1000 \mathrm{~K} \mathrm{~km} \mathrm{~s}^{-1}$ at the scale of GMCs (or larger). 


\section{More Active GCs: NGC 4418, Arp 220, and NGC 3256}

These three galaxies are infrared (ultra)luminous with $L_{\mathrm{TIR}} \geqslant 10^{11} L_{\odot}$. Two are late stage mergers. Galaxy collisions strongly perturb gas in galaxies and can cause more significant gas transport to the centers than bars do. Therefore the centers of merger-induced luminous galaxies are expected to show more extreme ISM properties and prominent interplay between molecular gas and the luminosity-generating activities.

Our SMA observations of the nearest ultraluminous infrared galaxy Arp 220 (Fig. 3) revealed bright compact peaks of $860 \mu \mathrm{m}$ continuum at the two merger nuclei in addition to confirming molecular gas peaks at and rotation around the individual nuclei. The brighter western nucleus has a $860 \mu \mathrm{m}$ continuum size of $\sim 50-80 \mathrm{pc}$ and a deconvolved brightness temperature about $100 \mathrm{~K}$, which sets a lower limit to the temperature of the dust emitting the continuum. The bolometric luminosity of the nucleus (i.e., its continuum core) has therefore a lower limit of $(2-3) \times 10^{11} L_{\odot}$ set by the size, temperature (lower limit), and the Stefan-Boltzmann law. The luminosity surface density of the continuum core is $\geqslant 10^{7.6} L_{\odot} \mathrm{pc}^{-2}$. Moreover, the bolometric luminosity-to-mass ratio of the nucleus is estimated to be $\geqslant 400 L_{\odot} / M_{\odot}$ by combining the luminosity with the dynamical mass of the nucleus estimated using the CO line velocities. These parameters, derived from the submillimeter high-resolution observations alone, suggest the energy source in the nucleus to be an AGN or a young ( $<10$ Myr) starburst (Sakamoto et al. 2008). We also found P-Cygni line profiles of molecular lines toward each nucleus from our SMA observations (Fig. 3 bottom). The blueshifted absorption lines suggest an outflow of molecular gas from each of the two nuclei. The outflows must be driven by the luminous active nuclei likely through radiation pressure on dust.

We can expect to have tighter constraints on the nature and ongoing evolution of both nuclei of Arp 220 and the nuclei of many luminous infrared galaxies from the submillimeter diagnostic methods used here. ALMA is going to observe these GCs at higher resolution, sensitivity, and accuracy as well as wider frequency coverage.

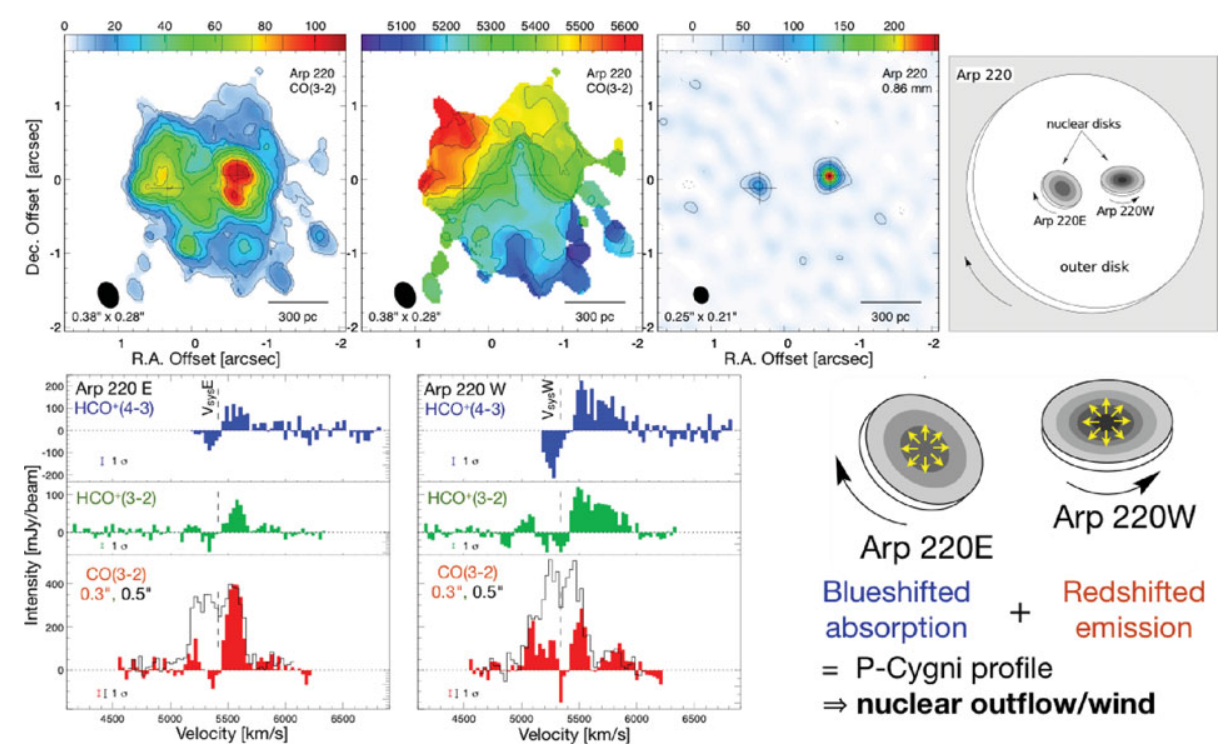

Figure 3. (Top) Submillimeter line and continuum images of Arp 220 from Sakamoto et al. (2008) and an illustration of the Arp 220 system from Sakamoto et al. (1999). (Bottom) Molecular line profiles toward the two nuclei (Sakamoto et al. 2009). The P-Cygni line profiles suggest an outflow of molecular gas from each nucleus. 


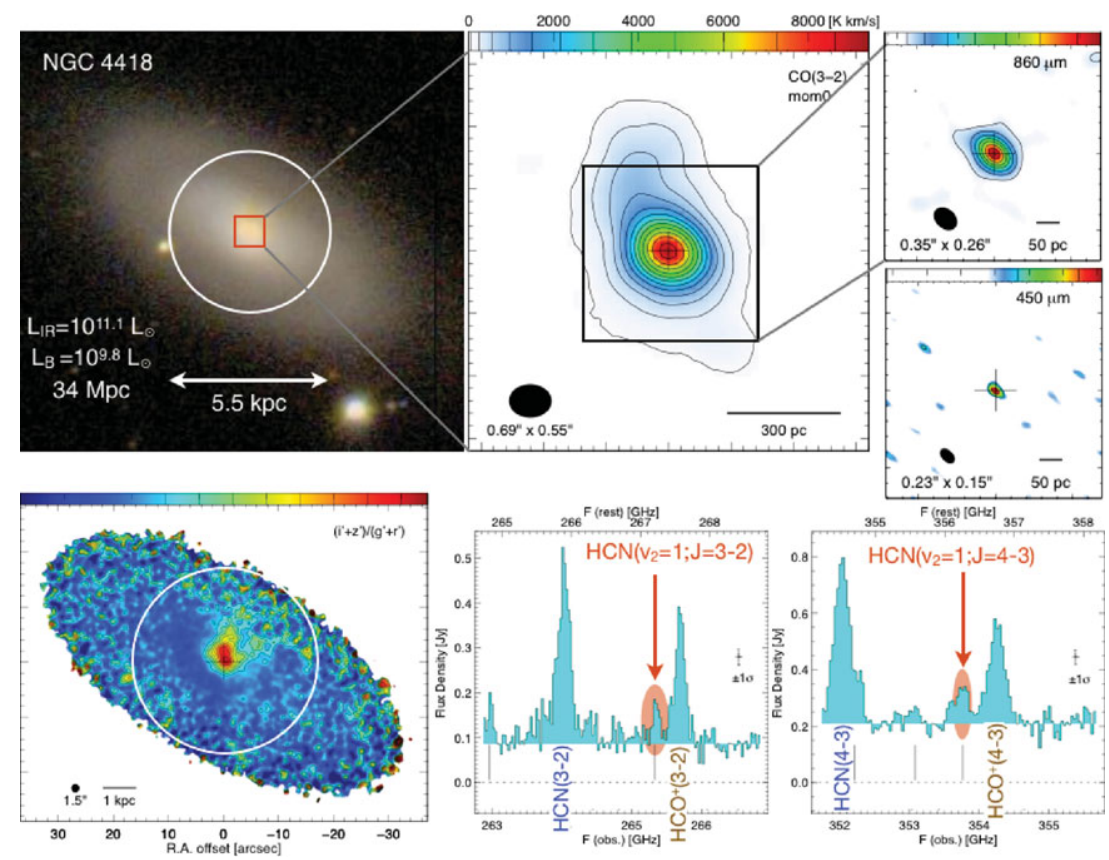

Figure 4. (Top, left to right) NGC4418 optical SDSS image, submillimeter CO(3-2) line map, and submillimeter continuum images. (Bottom left) Optical color index image showing a U-shaped outflow cone through reddening (Sakamoto et al. 2012b). (Bottom right) Spectra of the nucleus at $\lambda \sim 1 \mathrm{~mm}$ where rotational lines from vibrationally excited HCN were detected for the first time outside our Galaxy (Sakamoto et al. 2010).

We found in NGC 4418 a compact and hot concentration of molecular gas and dust as at Arp 220 nuclei (Fig. 4). This galaxy has infrared spectral features to suggest as heavy nuclear obscuration as in Arp 220 (Spoon et al. 2007) although NGC 4418 is not a major merger and is 10 times less luminous than Arp 220. The same analysis as we made for Arp 220 resulted in such parameters as $T_{\mathrm{b}} \geqslant 100 \mathrm{~K}, L_{\mathrm{bol}} \approx 10^{11} L_{\odot}, L_{\mathrm{bol}} / M_{\mathrm{dyn}} \geqslant 500$ $L_{\odot} / M_{\odot}$, and $N_{\mathrm{H}} \geqslant 10^{25} \mathrm{~cm}^{-2}$ for the nucleus (Sakamoto et al. 2012b). As in Arp 220, these parameters are consistent with an AGN being the dominant luminosity source while a young compact starburst is also possible since a starburst can have $L / M \approx 1000$ $L_{\odot} / M_{\odot}$ for several Myr. We also found a sign of outflow from the nucleus, this time from an optical color index map where a U-shaped reddening is on a semi-minor axis. Since Gonzáelz-Alfonso et al. (2012) found an inflow toward the nucleus from redshifted absorption lines, the nucleus of NGC 4418 has an outflow and an inflow in different directions. Such a gas flow structure has been modeled around a galaxy nucleus hosting both an AGN and starburst by Wada (this Symposium, and in Wada et al. 2012). We also detected rotational lines from vibrationally excited $\mathrm{HCN}$, from energy levels $E_{\text {up }} \approx 1000$ K, toward NGC 4418 nucleus for the first time in an external galaxy (Sakamoto et al. 2010). We estimated the vibrational excitation temperature to be about $230 \mathrm{~K}$. This detection clearly indicates that the nucleus is either very hot or has abundant infrared photon for radiative $\mathrm{HCN}$ excitation or both.

In the infrared luminous merger NGC 3256 we found a gas distribution similar to that in Arp 220 and high-velocity CO emission to suggest a molecular outflow (Fig. 5). This is the most luminous galaxy within $z=0.01\left(4 \times 10^{11} L_{\odot}\right)$. Its first interferometric $\mathrm{CO}$ imaging found twin concentrations of molecular gas at the merger nuclei (Sakamoto et al. 2006b). This is very similar to the two nuclear gas disks in Arp 220. We also found 


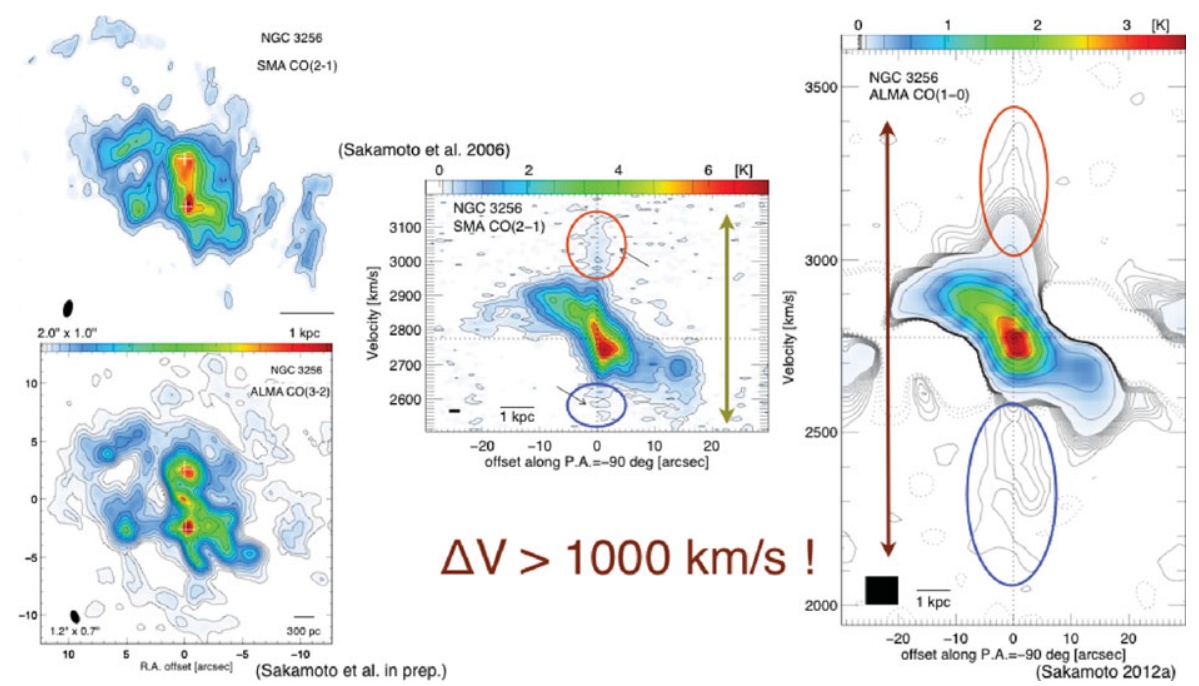

Figure 5. NGC 3256 observed with SMA and ALMA (Sakamoto et al. 2006b, 2012a, and in prep.). (Left) Molecular gas peaks at the two nuclei (white + ) and shows multiple spiral features around them. (Middle and Right) CO position-velocity diagrams across the merger center. The high-velocity CO emission that we first found with the SMA turned out to have a full width $>1000 \mathrm{~km} \mathrm{~s}^{-1}$ in the ALMA data.

high-velocity $\mathrm{CO}$ emission and attributed it to gas outflow from the galactic center. My collaborators and I have been following up these observations using ALMA for further details on the merger gas dynamics, feedback from the luminous nuclei, and comparison with Arp 220. So far we have confirmed the twin molecular gas peaks and started to see complex molecular spirals around the nuclei. Moreover, the high-sensitivity ALMA data show that the central CO line is much wider than in the SMA data. Vigorous energy generation and strong feedback to the surrounding ISM are apparently in action.

To summarize, SMA observations of nearby luminous galactic centers have revealed extreme ISM properties, such as high temperatures, excitations, and column densities. Signs of mechanical feedback are abundant in the forms of bubbles and outflows. A key subject to be studied with ALMA is the relation between these properties of molecular gas and dust and the energy generation activities in the GCs.

\section{References}

Elmegreen, B. G., Galliano, E., \& Alloin, D. 2009, ApJ, 703, 1297

Galliano, E., Kissler-Patig, M., Alloin, D., \& Telles, E. 2012, A\&A A, 545, A10

González-Alfonso, E., Fischer, J., Graciá-Carpio, J., et al. 2012, A\&\&A, 541, A4

Larson, R. B. 1981, MNRAS, 194, 809

Sakamoto, K., Ho, P. T. P., Iono, D., et al. 2006a, ApJ, 636, 685

Sakamoto, K., Ho, P. T. P., and Peck, A. B. 2006b, ApJ, 644, 862

Sakamoto, K., Ho, P. T. P., Mao, R.-Q., Matsushita, S., and Peck, A. B. 2007, ApJ, 654, 782

Sakamoto, K., Wang, J., Wiedner, M. C., et al. 2008, ApJ, 684, 957

Sakamoto, K., Aalto, S., Wilner, D. J., et al. 2009, ApJ, 700, L104

Sakamoto, K., Aalto, S., Evans A. S., Wiedner, M. C., and Wilner, D. J. 2010, ApJ, 725, L228

Sakamoto, K., Mao, R.-Q., Matsushita, S., et al. 2011, ApJ, 735, 19

Sakamoto, K. 2012a, arXiv:1207.3678

Sakamoto, K., Aalto, S., Costagliola, F. et al.. 2012b, submitted to ApJ .

Spoon, H. W. W., Marshall, J. A., Houck, J. R., et al. 2007, ApJ, 654, L49

Wada, K. 2012, ApJ, 758, 66 